\title{
Migration and Thermotaxis of Dictyostelium discoideum Slugs, a Model Study
}

\author{
Athanasius F.M. Marée,* Alexander V. Panfilov and Paulien Hogeweg \\ Department of Theoretical Biology, University of Utrecht, Padualaan 8, 3584 CH Utrecht, \\ The Netherlands
}

(Received on 16 October 1998, Accepted in revised form on 20 April 1999)

\begin{abstract}
Dictyostelium discoideum slugs show a pronounced thermotaxis. We have modelled the motion of the D. discoideum slug in the absence and in the presence of a thermal gradient. Our model is an extension of the hybrid cellular automata/partial differential equation model, as formulated by Savill and Hogeweg [J. theor. Biol., (1997) 184, 229-235]. The modelled slugs maintain their shape and crawl, with a velocity depending on slug size, as found in experiments. Moreover, they show thermotactic behaviour: independent of the initial orientation, after some transient process, the slugs start moving along the temperature gradient. The slug behaviour in our model is due to the collective behaviour of the amoebae. Individual amoebae can neither respond to a shallow temperature gradient, nor show differentiation in motion velocity. The behaviour is achieved by a modification of the cyclic AMP waves: differences in temperature alter the excitability of the cell, and thereby the shape of the cyclic AMP wave. Chemotaxis towards cyclic AMP causes the slug to turn. We show that the mechanism still functions at very low signal-to-noise ratios.
\end{abstract}

(C) 1999 Academic Press

\section{Introduction}

The cellular slime mould Dictyostelium discoideum is a soil protozoan that feeds on bacteria. This model organism has been extensively studied, as the life cycle of the organism provides a unique opportunity to study the relation between signal transduction at the cellular level and morphogenesis and behaviour at the multicellular level. Upon starvation, individual amoebae aggregate and form migrating multicellular slugs. During aggregation the amoebae differentiate into prestalk and prespore cells. The prestalk cells group into the front part, while the rear part consists of prespore cells. Each slug culminates

\footnotetext{
*Author to whom correspondence should be addressed. E-mail: stan@binf.bio.uu.nl.
}

in a fruiting body consisting of a globule of spore cells on a slender stalk.

The aggregation is orchestrated by waves of cyclic AMP (cAMP), which are formed by a combination of a pulsatile cAMP excretion and a cAMP-mediated cAMP response; the amoebae show a chemotactic response towards cAMP. This combination of waves of excitation and chemotaxis persists during the slug stage.

Migrating slugs are oriented by temperature gradients. This thermotaxis shows a significant temperature adaptation, with positive thermotaxis at temperatures above the temperature-during aggregation, and negative thermotaxis below this temperature (Whitaker \& Poff, 1980). At night, the soil surface is cooler than the subsurface mulch, and hence the slug is directed by 
negative thermotaxis towards the surface. At daytime the reverse is true, but the slug still moves upwards, this time due to positive thermotaxis. As a result, the slug always tends to migrate towards the soil surface where it will fruit, thus ensuring good conditions for spore dispersal (Bonner et al., 1985).

How a temperature gradient is converted into tactic behaviour is not yet fully understood, but several researchers have shown that ammonia $\left(\mathrm{NH}_{3}\right)$ plays an important role in this process, since temperature influences $\mathrm{NH}_{3}$ production significantly, and thermotaxis diminishes when slugs are surrounded by $\mathrm{NH}_{3}$ (Bonner et al., 1989). Three roles of $\mathrm{NH}_{3}$ have been proposed. First, thermotaxis may be due to negative chemotaxis away from $\mathrm{NH}_{3}$. Several authors have reported such negative chemotaxis, and have shown that a slug can produce sufficient concentrations of $\mathrm{NH}_{3}$ to account for such an orientation (Bonner et al., 1986; Feit \& Sollitto, 1987; Kosugi \& Inouye, 1989; Yumura et al., 1992).

A second role of $\mathrm{NH}_{3}$ may be that it speeds up cell motion. Bonner et al. (1986) reported such an increase of cell motion due to $\mathrm{NH}_{3}$. Based on this result, a model was constructed for thermotaxis (Bonner et al., 1989). However, this report is still disputed. van Duijn \& Inouye (1991) found that $\mathrm{NH}_{3}$ only slightly, non-significantly, increased the chemotactic-locomotion speed. In fact, more detailed studies reported that average slug speed may be unaffected by $\mathrm{NH}_{3}$ or temperature gradients (Smith et al., 1982; Fisher, 1997). Furthermore, Davies et al. (1993) reported that $\mathrm{NH}_{3}$ did not cause any change in chemotaxis towards cAMP.

Third, $\mathrm{NH}_{3}$ may affect the cAMP signalling. Schindler \& Sussman (1979) showed that $\mathrm{NH}_{3}$ inhibits the cAMP-induced cAMP release. The underlying mechanism is that $\mathrm{NH}_{3}$ blocks the intracellular cAMP accumulation, due to the inhibition of the transitory activation of adenylate cyclase in response to the binding of extracellular cAMP to cell surface receptors (Williams et al., 1984; Davies et al., 1993). Furthermore, Darcy \& Fisher (1990) produced evidence that this inhibition of the cAMP signalling is important in slug behaviour.

We use only the latter role of $\mathrm{NH}_{3}$ in our model. As a shortcut, we model the inhibition of the cAMP relay by $\mathrm{NH}_{3}$ as a direct (negative) influence of temperature on the excitability.

There have been several models describing slug migration (Odell \& Bonner, 1986; Williams et al., 1986; Umeda, 1989; Bretschneider et al., 1995; Savill \& Hogeweg, 1997; Dormann et al., 1998). However, until now no one has used a model for slug migration to describe thermotaxis. The purpose of this study, therefore, was to determine whether thermotaxis can be achieved using basic and accepted principles of $D$. discoideum information processing at the cellular level. To study this topic, we used the model of Savill \& Hogeweg (1997) because it handles pressure, deformation and motion in a very elegant way, and enables us to investigate thermotaxis in the context of the complete development from single cells to fruiting bodies. The model of Savill \& Hogeweg (1997) is three-dimensional (3D), but for our purposes we can use two-dimensional (2D) simulations. Besides, very recently, Bonner (1998) presented a new method in which migrating 2D (one cell thick) slugs are produced at a glassmineral oil interface. This creates the opportunity to compare our results directly with the experimental findings of Bonner.

In our model, thermotaxis is achieved by a modification of the cAMP waves, due to the differences in the excitability. These differences change the shape of the wave, and chemotaxis towards the wave leads to the tactic behaviour.

\section{The Model}

We have used the hybrid cellular automata (CA)/partial differential equation (PDE) model of Savill \& Hogeweg (1997), which is based on the CA model of Glazier \& Graner (1993). In this model, a CA is used to represent individual amoeba, and a PDE to model the cAMP dynamics. An important feature of the model is that each amoeba is represented as a group of connected automata. As a consequence, amoebae can slide past one another and deform themselves and the adjoining amoebae by means of small changes in their boundaries.

\subsection{DESCRIPTION OF THE AMOEBAE}

The space where the slug crawls is a $2 \mathrm{D}$ lattice. Each lattice square represents an automaton as 
well as a grid point in the numerical PDE. Each amoeba has a unique identification number, $\sigma$, which is assigned to all automata which form the amoeba. $\sigma=0$ represents the medium. Amoebae also have a label $\tau$, which indicates whether their cell type is prespore, prestalk or autocycling prestalk $(\tau \in\{p, t, a\})$.

Each automaton that is part of an amoeba's boundary, i.e. for which one of its eight neighbouring automata does not belong to the same amoeba, has dimensionless free energy bonds. The magnitude of these bonds depends on the cell types they connect. The energy bonds are given by $J_{\tau_{1}, \tau_{2}}>0$, where $\tau_{i}$ are the types of the adjoining amoebae. The bond energy between an amoeba and the medium is given by $J_{\tau, M}$. The total free energy of an amoeba is given by

$$
\begin{aligned}
H_{\sigma}= & \sum \frac{J_{\text {cell }, \text { cell }}}{2} \\
& +\sum J_{\text {cell }, \text { medium }}+\lambda(v-V)^{2},
\end{aligned}
$$

where $v$ is the volume of the cell, $V$ the target volume, and $\lambda$ the inelasticity. The final term ensures that the volume of a cell remains close to $V$.

In order to model an amoeba deforming its shape, we use the following rule: an automaton is chosen at random and the state of one of its neighbours is copied into it with a probability of occurring given by

$$
\begin{aligned}
& \Delta H<-0.8, \quad \text { Prob }=1, \\
& \Delta H \geqq-0.8, \quad \text { Prob }=e^{-(\Delta H+0.8 / T)},
\end{aligned}
$$

where $\Delta H$ is the change in energy if the copying were to occur, and $T$ the default mobility of the amoebae. These equations model minimization of the amoeba's free energy. We chose bond energies so that the amoebae adhere to each other, and also sort themselves into three fairly homogeneous groups, if it were possible. Autocycling cells adhere to each other more rigidly than prestalk cells do, and the latter adhere to each other more rigidly than prespore cells do.

\subsection{CAMP DYNAMICS}

The cAMP signalling system is rather complex and can be described using detailed models, such as, e.g. Martiel \& Goldbeter (1987) and Tang \& Othmer (1994). However, the dynamics can be described reasonably well in a quantitative way by two variable simplified equations of the FitzHugh-Nagumo (FHN) type (see e.g. Grindrod, 1996). Such a description reproduces the overall characteristics of cAMP waves such as refractoriness, eikonal-curvature relation, etc. The main advantage of these models is their simplicity and therefore their capacity to connect the effects visible in these models with basic properties of the cAMP signalling in D. discoideum.

Here we investigate the basic effects of heterogeneity in the excitability, but we are not concerned with the details of cAMP signalling. So for our present purposes, the FHN-models are preferred. In this paper, we use FHN-type equations with piecewise linear "Pushchino kinetics" (Panfilov \& Pertsov, 1984). This system allows for even greater control of the refractory periods and is much faster to compute than the classical system (Panfilov, 1991):

$$
\left.\begin{array}{l}
\frac{\partial c}{\partial t}=D_{c} \Delta c-f(c)-r \\
\frac{\partial r}{\partial t}=\varepsilon(c)(k c-r)
\end{array}\right\} \text { inside the amoebae }
$$

with $f(c)=C_{1} c$ when $c<c_{1} ; f(c)=-C_{2} c+a_{\tau}$ when $c_{1} \leqslant c \leqslant c_{2} ; f(c)=C_{3}(c-1)$ when $c>c_{2}$, and $\varepsilon(c)=\varepsilon_{1}$ when $c<c_{1} ; \quad \varepsilon(c)=\varepsilon_{2}$ when $c_{1} \leqslant c \leqslant c_{2}$ and $\varepsilon(c)=\varepsilon_{3}$ when $c>c_{2}$. To make the function $f(c)$ continuous, $c_{1}=a_{\tau} /\left(C_{1}+C_{2}\right)$ and $c_{2}=\left(a_{\tau}+C_{3}\right) /\left(C_{2}+C_{3}\right) \cdot c$ represents cAMP concentration, and $r$ the refractoriness of the cells. Outside the amoebae a small decay $d_{c}$ of cAMP, caused by external phosphodiesterase, is implemented. The arbitrary zero level $c_{0}$ is chosen such that cAMP leakage into the medium does not decrease the period of autocycling.

Weijer et al. (1984) showed that the tip of the slug can be seen as a high-frequency pacemaker, whereas the body of the slug just conducts waves of cAMP. We can model this in the following way. For positive values of parameter $a_{\tau}$, the model describes an excitable medium, while for 
negative values of $a_{\tau}$, a stable limit cycle appears in phase space. Therefore, to describe the excitable amoebae, we have chosen $a_{p}=a_{t}=0.1$, whereas to describe the autocycling amoebae in the tip, $a_{a}=-0.1$.

To illustrate these basic properties of the model, we plot in Fig. 1 the phase plane of $c$ and $r$. Figure 1(a) shows the situation when $a_{\tau}=$ -0.1 . The nullclines intersect at an unstable equilibrium point, and the dynamics show a stable limit cycle. The inset shows the oscillatory behaviour in time. Figure 1(b) shows the phase plane when $a_{\tau}=0.1$. Now the equilibrium point is stable, and the system relays a cAMP signal if the initial concentration of cAMP exceeds the threshold value.

We simplify the whole process of differential $\mathrm{NH}_{3}$ production due to the temperature gradient, followed by differential inhibition of the cAMP-modulated cAMP response due to $\mathrm{NH}_{3}$, by stating that the temperature gradient causes a spatial gradient in the excitability. We have chosen to model the differences in the excitability by changing the value of $a_{\tau}$, since in this model $a_{\tau}$ represents the threshold for cAMP relay, which is directly related to the excitability. We superimpose on the values of $a_{\tau}$ a gradient along the $y$-axis, with a slope of $a_{\text {grad }}$ per grid point, and with decreasing excitability from top to bottom. We normalize the gradient by taking the upmost automaton that is occupied by the slug as the reference point to calculate all $a_{\tau}$ values. We use a normalized gradient to assure that the behaviour is independent of the location of the slug in the field.

\subsection{CHEMOTAXIS}

The natural way to incorporate chemotaxis in the model is to use the spatial gradient of the cAMP wavefront. We do this by modifying the change in energy $\Delta H$ in eqn (2), and hence the probability of the neighbour copying its state into the automaton, as a function of the difference in the cAMP concentrations in the two automata (Savill \& Hogeweg, 1997):

$$
\Delta H^{\prime}=\Delta H-\mu\left(c_{\text {automaton }}-c_{\text {neighbour }}\right),
$$

where $\Delta H^{\prime}$ is the new change in energy. This equation implies that an amoeba is more likely to move to an area with a higher cAMP concentration and less likely to move to an area with a lower cAMP concentration. All amoebae obey this rule and $\mu$ is invariant over all amoebae.
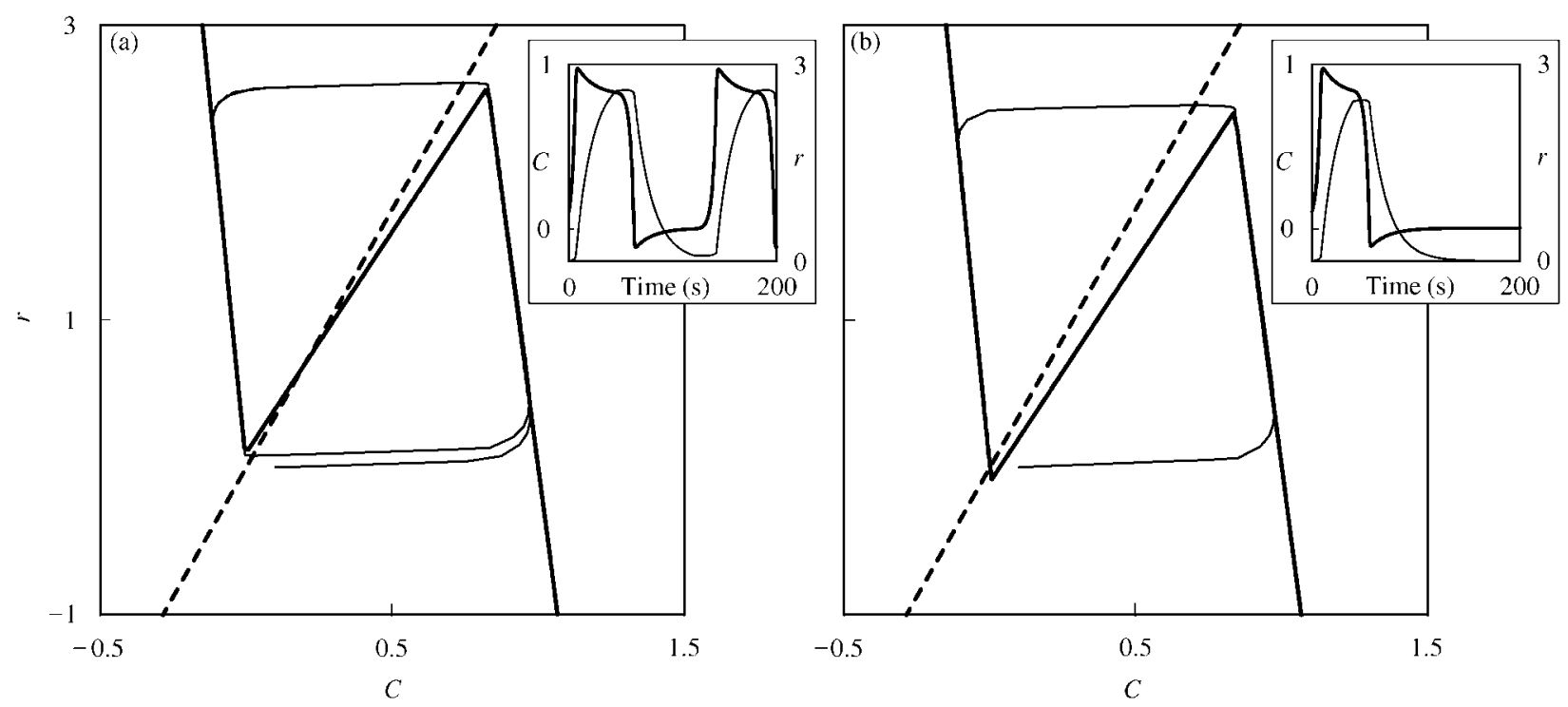

FIG. 1. Phase plane of $c$ and $r$ for (a) $a_{\tau}=-0.1$; and (b) $a_{\tau}=0.1$. The thick solid lines denote the $\mathrm{d} c / \mathrm{d} t=0$ nullclines, the thick dashed lines denote the $\mathrm{d} r / \mathrm{d} t=0$ nullclines. The thin lines depict trajectories starting from $c=0.1$ and $r=0$. The insets show the corresponding behaviour of $c$ (thick lines) and $r$ (thin lines) in time. Parameters: $C_{1}=20, C_{2}=3, C_{3}=15, \varepsilon_{1}=0.5$, $\varepsilon_{2}=0.0589, \varepsilon_{3}=0.5$, and $k=3.5$. 
Since the amoebae only move chemotactically during a short period after arrival of the cAMP wave, rule (5) is only applied when $c$ is above a threshold $c_{t h}=0.05$ and $r$ below a threshold $r_{t h}=0.2$.

\section{Slug Migration}

In all simulations, a slug is initiated with a tip consisting of autocycling prestalk cells, and a body consisting of $40 \%$ prestalk and $60 \%$ prespore cells.

Figure 2(a) shows slug motion in a simulation without thermotaxis. Repeatedly the chemotactic signal spreads from the autocycling cells in the tip towards the posterior part of the slug. The subsequent chemotactic motion pushes the tip forward, and as a result the whole slug moves ahead. We see that the slug does not follow a straight line. Its trail shows alterations in direction, caused by small variations in the way the tip is pushed forward.

\subsection{STABILITY OF THE SLUG SHAPE}

As can be seen in Fig. 2, during the simulations, which take up 300000 time-steps $(8 \mathrm{~h}$ and $20 \mathrm{~min}$ ), the shape of the slug is approximately fixed. This stable configuration results from two forces; one elongates the slug, and the other one rounds it. The surface tension forces the slug to round, since this is the optimal low-energy configuration, whereas the convex-shaped cAMP wave causes a motion of the amoebae towards the central axis, and therefore forces the slug to elongate.

Different surface tensions lead to different slug shapes. Hence, we chose the values of $J_{\tau, M}$ so that the surface tension with the medium, which is defined by Glazier \& Graner (1993) as $J_{\tau, M}-1 / 2 J_{\tau, \tau}$, is equal for all cell types.

The convex shape of the cAMP wave is caused by two factors: first, every new cAMP wave originates as a circular wave from a point source, which immediately leads to a convex wave shape. Secondly, the speed of the cAMP wave is lower at the slug boundary, because amoebae along the boundary of the slug are less excitable, due to loss of cAMP into the surrounding medium.
In our computations, we have chosen an initial shape which is close to the final shape of the slug. By starting with another shape, which is thinner or thicker than the optimal shape, one adds extra transient behaviour, but the final configuration is the same.

\subsection{MOTION VELOCITY}

Figure 3 shows the relation between the slug mass and the motion velocity. To measure motion velocity, tracks of slugs were followed during $5.5 \mathrm{~h}$ (200000 time-steps). We see that if a slug consists of 20 amoebae, velocity is only $50 \%$ of the maximum velocity, while larger slugs clearly move faster. This increase in motion velocity seems to saturate when the number of amoebae gets close to 1000 . Our results are consistent with the experimental data: it is known from experiments that the slug velocity increases with increase in the slug size (Bonner et al., 1953; Smith et al., 1982), and Inouye \& Takeuchi (1979) showed the saturation of velocity increase for large slugs.

The velocity increase we found is on the one hand due to the fact that more amoebae create more motive force to push the autocycling area forward, since this region does not create a motive force by itself. On the other hand, to the fact that the motive force created by the slug does not solely depend on chemotaxis towards cAMP. Also the accompanying volume changes, and the binding strengths as well as the surface tension play an important role: First, due to the binding forces between the amoebae, amoebae start moving before the cAMP wave arrives, as they are pulled towards the chemotactically moving amoebae. Second, the surface tension creates a forward motion to fill up the dents in the slug shape just posterior of the cAMP wave. Third, due to volume decrease during the chemotactic motion, as many cells push inwards, motion lasts after the cAMP wave has passed, to re-establish the old volume. Since all these depend on the slug size, the motion is faster when the slug is larger.

The saturation in velocity increase with size, as described by Inouye \& Takeuchi (1979), occurs in slugs with much larger number of amoebae than in our finding. This can be caused by the fact that we performed $2 \mathrm{D}$ simulations, and due to scaling reasons. 


\subsection{CELL SORTING}

It has been known for some time that cell sorting takes place mainly during the mound stage that precedes the slug stage. However, both dissociation experiments (Sternfeld \& David, 1981) and transplantation experiments (MacWilliams, 1982) have shown that slugs are still fully capable of exhibiting cell sorting.

In our simulations we start from a random distribution of prestalk and prepore cells, and after $4 \mathrm{~h}$, prestalk and prespore cells are completely sorted out. In our model, this cell sorting is solely due to differential adhesion, since there are no other differences between the cell types. When $J_{\tau, \tau}$ is smaller, amoebae with the same inelasticity $\lambda$ deform less, since the final term in eqn (2), which ensures volume conservation, is relatively more important. As a consequence, the extra motive force of pulling the cells forward is larger. In contrast, when $J_{\tau, \tau}$ is larger, the amoebae just deform and hence have a relatively slower motive speed. Thus, to obtain autocycling cells in the tip, followed by prestalk cells, and finally prespore cells, $J_{a, a}<J_{t, t}<J_{p, p}$. The binding energies between the cell types are less important for cell sorting. They are kept high enough to assure that the cell types do not mix, and are kept low enough to assure that amoebae of different cell types are still able to slide past one another, which is necessary for large-scale cell sorting.

In our simulations, if a clump of autocycling cells are positioned closely behind the tip, these cells become entrained to the cAMP signalling from the tip, and moreover, after some time, by the process of cell sorting as described above, the clump reunites with the autocycling cells in the tip (results not shown). Only if either the clump or the difference in the excitability with the surrounding cells is large enough, these cells themselves become a new source of cAMP signalling, which, after some time, results in the break up of the slug into two small ones.

\section{Thermotaxis}

We were able to reproduce thermotaxis of the D. discoideum slug. Figure 2(b) shows slug motion in a field with a temperature gradient directed from top to bottom. Initially the slug is oriented at an angle of $90^{\circ}$ to the direction of the gradient. Yet within $3 \mathrm{~h}$ the slug orients itself towards the gradient.

Figure 4 shows the tracks of $D$. discoideum slugs from 25 different simulations, with initial angles of $0,45,90,135$, and $180^{\circ}$ to the temperature gradient. It can be seen that the final direction of motion does not depend on the initial angle. Even if the initial angle is $180^{\circ}$, standard irregularities in the track cause small deviations in the angle that are large enough to elicit the subsequent turn, either to the left or to the right.

\subsection{MECHANISM OF THERMOSENSITIVITY}

In our model, we assume a temperature dependence of the cAMP-induced cAMP release (presumably mediated by $\mathrm{NH}_{3}$ ). We implement this as a change in the excitability, i.e. by a modification of the value of the $a_{\tau}$ parameter. Hence, we change the cAMP threshold. An increased threshold changes both the period of autocycling and the velocity of the cAMP wave. Figure 5(a) shows the dependence of the period on $a$ in the oscillatory regime, i.e. for negative values of $a$. Figure 5(b) shows the dependence of the velocity on $a$ in the excitable regime, i.e. for positive

FIG. 2. Time sequences from simulations of motion during the slug stage of D. discoideum. (a) Motion without a temperature gradient. (b) Simulation of thermotaxis with a temperature gradient from top to bottom. (c) Simulation of thermotaxis with Gaussian noise on the $a_{\tau}$ parameter at every grid point, with a refreshment rate of $2 \mathrm{~s}$ (20 time-steps). (d) Simulation of thermotaxis with Gaussian noise on the level of the amoebae. The first frame in (a) shows the initial distribution for all four simulations; the first frames in (b), (c) and (d) show the state after $30 \mathrm{~min}$ (18 000 time-steps). Successive frames with intervals of $1 \mathrm{~h}$ (36000 time-steps). The axes are in grid points. There are 430 amoebae. One time step (solution of the PDE) corresponds to about $0.1 \mathrm{~s}$ and one grid point to $5 \mu \mathrm{m}$. Cell types are $\tau \in\{a, t, p\}$ where $a$ is autocycling prestalk (blue), $t$ is prestalk (red), and $p$ is prespore (green). Bond energies are $J_{a, a}=3, J_{t, t}=5, J_{p, p}=7, J_{a, M}=7, J_{t, M}=8, J_{p, M}=9, J_{a, t}=6, J_{t, p}=8, J_{a, p}=9$. $T=2, V=30, \lambda=0.6$ and $\mu=200$. The parameters used for the PDE equations are $D_{c}=1, c_{0}=-0.3, d_{c}=0.05$, and $a_{\text {grad }}=6 \times 10^{-4}$. Other parameters are as described in the legend to Fig. 1. Gaussian noise with mean $=0$ and S.D. $=0.02$. The PDE equations are solved by the explicit Euler method (with time step equal to 0.01 and space step equal to 0.37 ). 


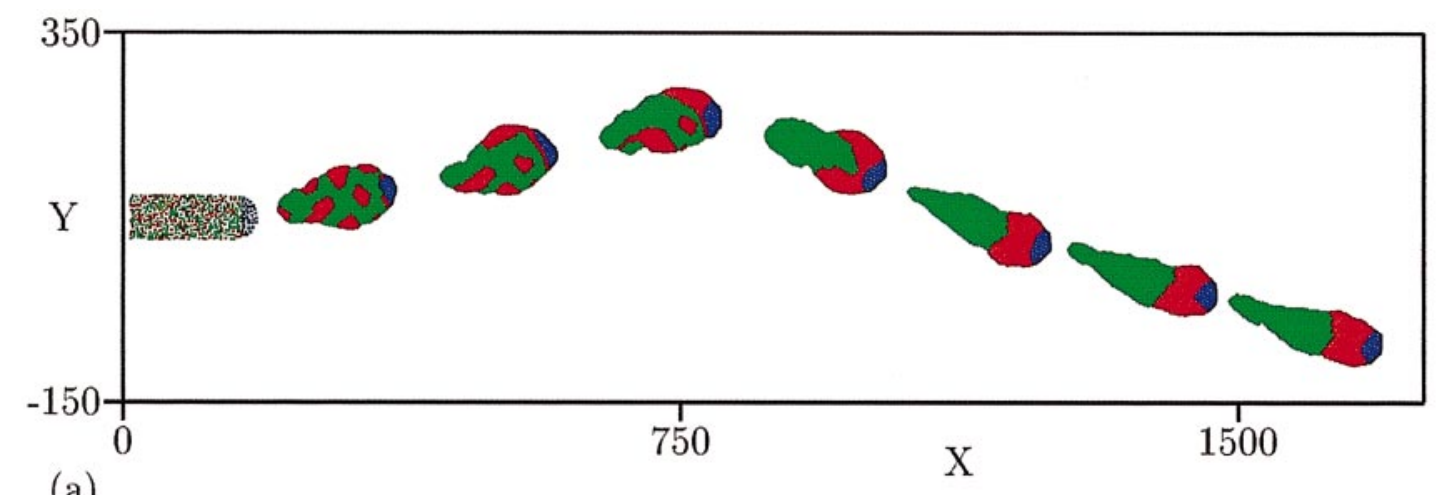

(a)
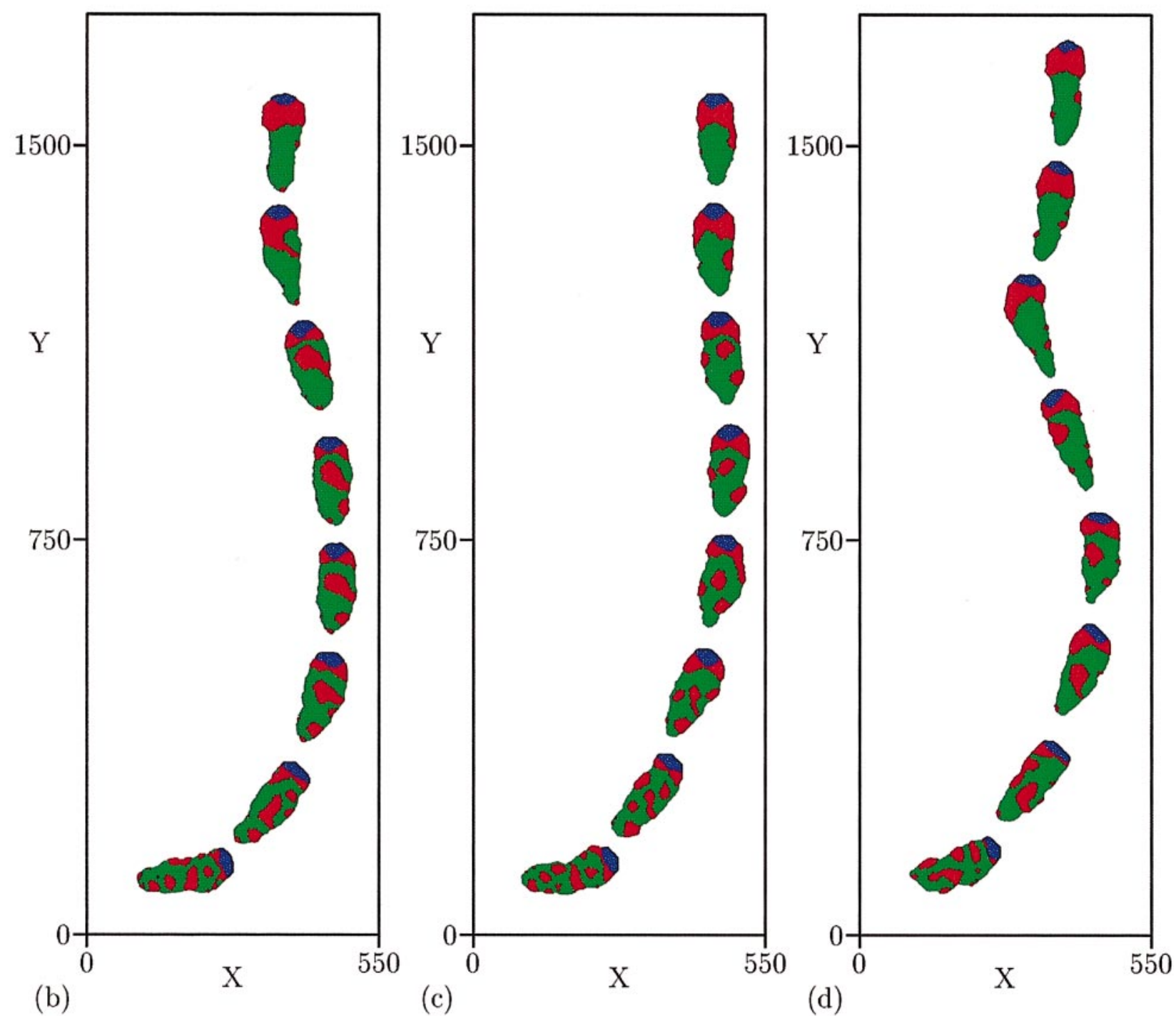

(Caption on page 302) 


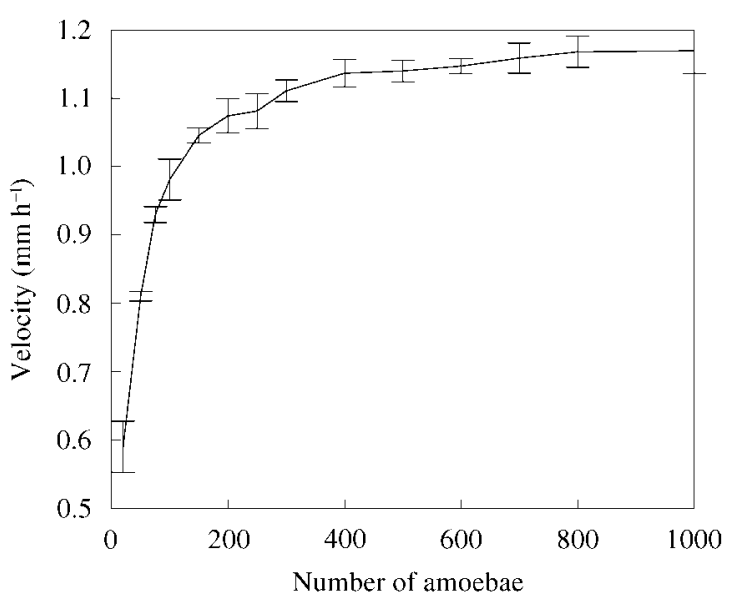

FIG. 3. Plot of the velocity of the slug versus the number of amoebae during non-thermotactic motion. The means and S.E.M. (indicated by the bars) of five simulations per data point are presented: $1 \mathrm{~mm} \mathrm{~h}^{-1}$ equals 0.0056 grid point per time-step. All parameters are as described in the legend to Fig. 2(a).

values of $a$ (note that in the oscillatory regime wave velocity depends on both period and relay). For reasonable values of $a$ the period increases with increasing $a$, and over the whole range of values the velocity decreases. Both are important for the mechanism of thermotaxis as they influence both the source of the cAMP signal and the shape of the cAMP wave.

Figure 6 shows a close up of the cAMP wave. Figure 6(a) shows the cAMP wave during normal motion, while Fig. 6(b) shows the wave during thermotaxis. To emphasize the effect of the temperature gradient on the shape of the cAMP wave, we have chosen a rather high value of $a_{\text {grad }}=2.5 \times 10^{-3}$. During thermotaxis, the source of the signal in the autocycling area shifts upwards, since in the upper direction $a$ values are lower, and subsequently the autocycling period is shorter. Besides, as a result of the dependence of wave speed on $a$, the wave moves faster at the upper side compared to the lower side. As a consequence of both, the wavefront becomes slanted. However, the slant increases only up to a certain level, since it coincides with a curvature difference that limits the slant, due to the so-called curvature effect.

Since the amoebae move perpendicular to the wavefront, a motive force is created that pushes the autocycling area upwards, and with it the

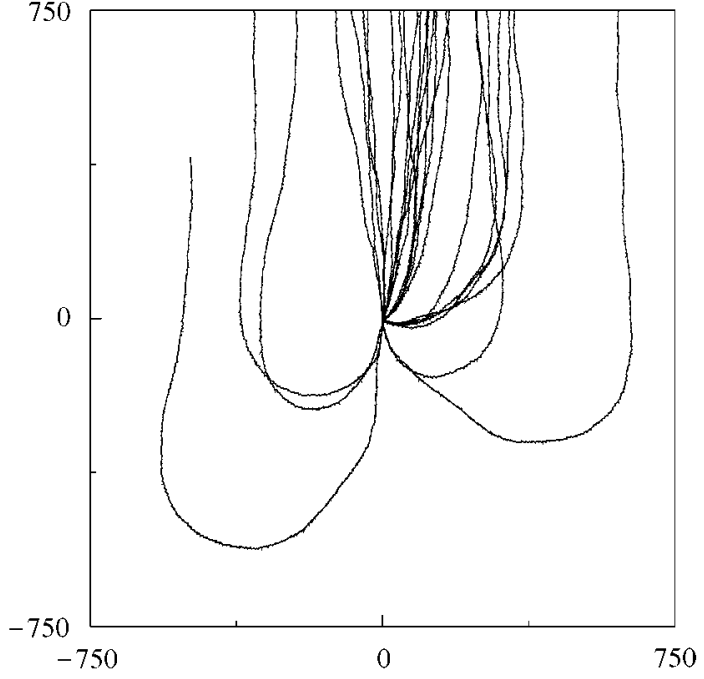

FIG. 4. Change in the slug location in time, during $8 \mathrm{~h}$ and $20 \mathrm{~min}$ (300000 time-steps). The location at $t=0$ is set to $(0,0)$, and the axes are in grid points. 25 simulations are presented, at an initial angle of $0,45,90,135$, or $180^{\circ}$, and with five simulations per angle. $a_{\text {grad }}=1.2 \cdot 10^{-3}$, all other parameters are as described in the legend to Fig. 2(b).

source of the cAMP signal, thus initiating the turn towards the temperature gradient.

\subsection{THERMOSENSITIVITY AND THE INFLUENCE OF STOCHASTIC NOISE}

We studied thermosensitivity as a function of $a_{\text {grad }}$. Figure 7 shows the tracks of slugs from simulations with different gradient strengths. In all simulations, the initial angle is $90^{\circ}$ to the temperature gradient. In Fig. 7(a) and (b), we see that when the gradient is very steep, the slugs show a rapid turn towards the temperature gradient. After this turn, the track is very stable and perfectly aligned along the temperature gradient. In Fig. 7(c) and (d), we see that when the gradient becomes more shallow, turning of the model slug takes increasingly more time, and the final track becomes more and more unstable. In Fig. 7(e), the gradient is as small as $a_{\text {grad }}=1.5 \times 10^{-4}$. Although the slugs do not move along the temperature gradient, they still move in the right direction. Note that we even found thermosensitivity for much smaller $a_{\text {grad }}$, i.e. $a_{\text {grad }}=6 \times 10^{-5}$.

However, in an environment with such shallow gradients, one expects to find a relatively high level of disturbance on the signal. Besides, there 

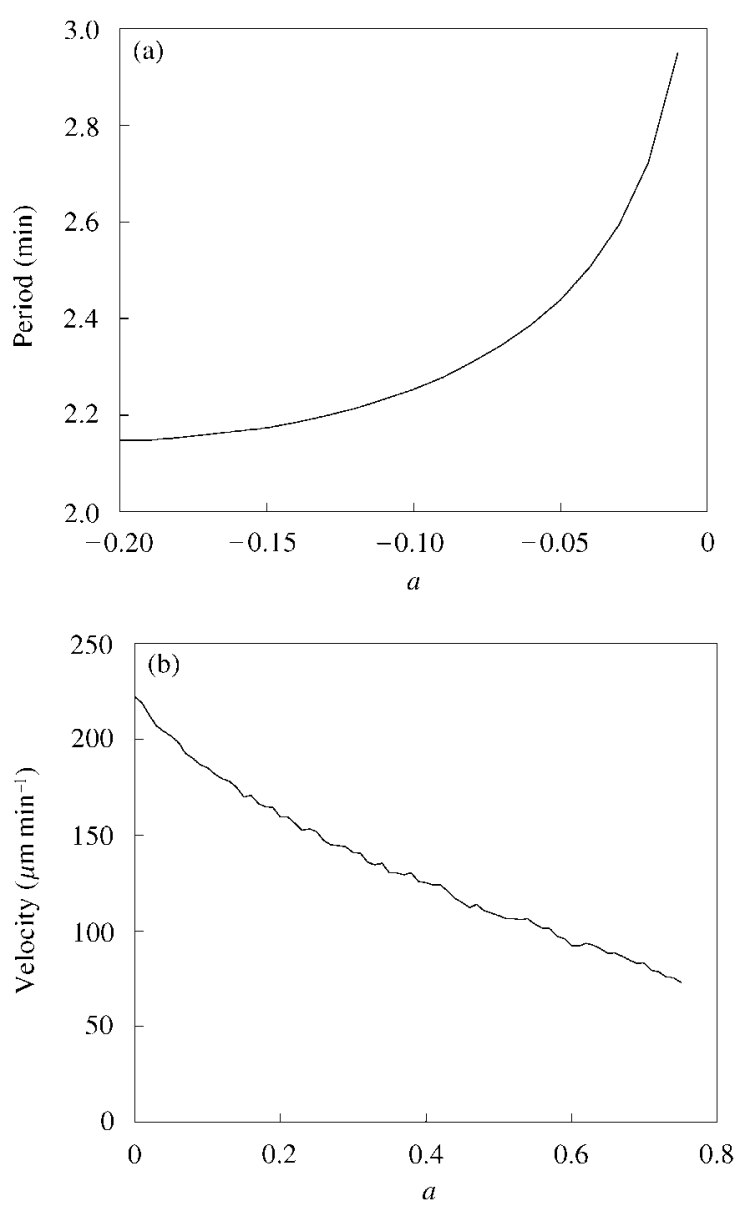

FIG. 5. Plot of the autocycling period in the oscillatory regime (a), and the velocity of the cAMP wave in the excitable regime (b), vs. parameter $a$. The period increases, while the velocity decreases with increasing $a$. 1 min equals 600 time-steps and $100 \mu \mathrm{m} \mathrm{min}^{-1}$ equals 0.033 grid point per time-step.

must exist some variation in the excitability between individual amoebae. Therefore, an important question is whether an addition of noise to the values of $a_{\tau}$ reduces or diminishes thermotaxis.

To test the sensitivity to disturbances, spatiotemporal Gaussian noise is added to the value of $a_{\tau}$, with a refreshment rate of $2 \mathrm{~s}$ (20 time-steps). We expect that in real slugs disturbances are high, relative to the shallow gradients at which a $D$. discoideum slug still exhibits thermotaxis. Therefore, we use a high noise term, with a normal distribution of mean $=0$, and S.D. $=0.02$, i.e. more than 30 times as large as $a_{\text {grad }}$. Figure 8 gives an impression of the signal-to-noise ratio for a certain moment in time. The ordinate de-
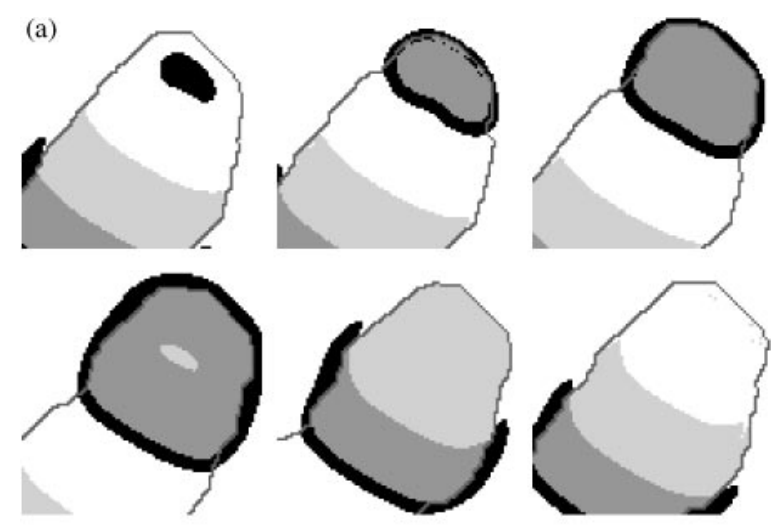

(b)
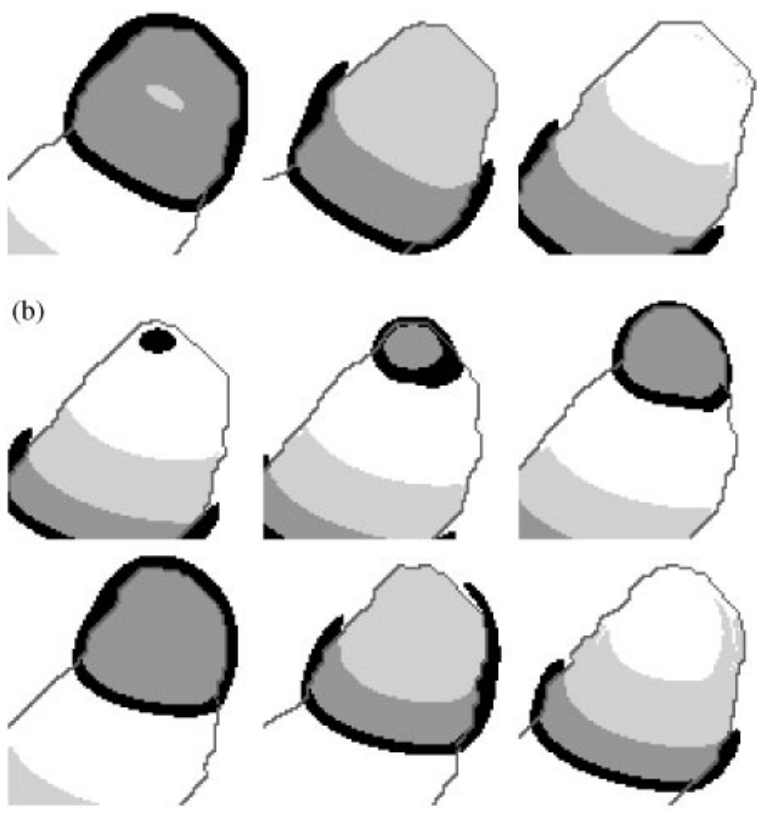

FIG. 6. Propagation of the cAMP wave: (a) during normal motion; and (b) during thermotaxis. During thermotaxis, the wave starts at a position shifted into the upper direction, and is slanted to the left. Successive frames after $15 \mathrm{~s}, 30 \mathrm{~s}, 1 \mathrm{~min}, 1 \frac{1}{2} \mathrm{~min}$, and $2 \mathrm{~min}(150,300,600,900$, and 1200 time-steps). Black denotes the area where $c>c_{t h}$ and $r<r_{t h}$ (the wavefront); dark grey the area where $c>c_{t h}$ and $r>r_{t h}$ (the waveback); and light grey represents the area where $c<c_{t h}$ and $r>r_{t h}$ (the refractory period). $a_{\text {grad }}=2.5 \times 10^{-3}$, all other parameters are as described in the legend to Fig. 2(a) and (b).

notes the location of the slug in the $y$-direction, the abscissa gives the corresponding increase of $a_{\tau}$. The solid line shows the gradient in the absence of noise. The dots show the increase of $a_{\tau}$ for all individual automata in the presence of noise. Note that the gradient is very difficult to detect when noise is present. Figure 2(c) shows a simulation with this spatio-temporal noise. In spite of the high level of noise, both precision and turning velocity are not decreased. In fact, noise levels have to be increased more than ten times before thermotaxis diminishes.

Secondly, we create heterogeneity in the amoebae population. The same level of variation 
(a)

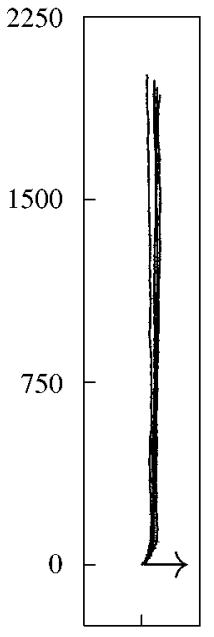

0 (b)

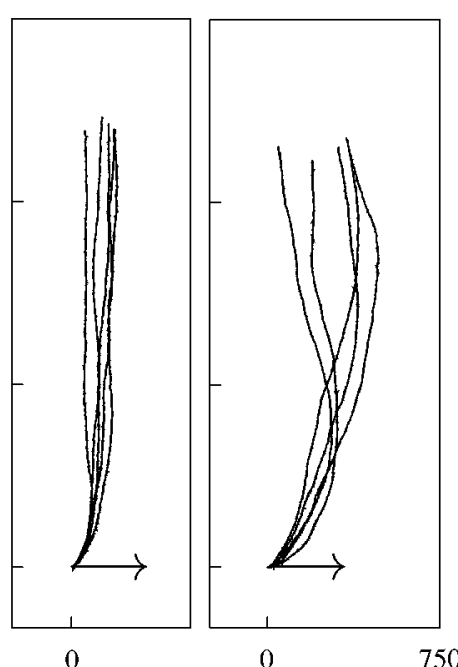

(d)

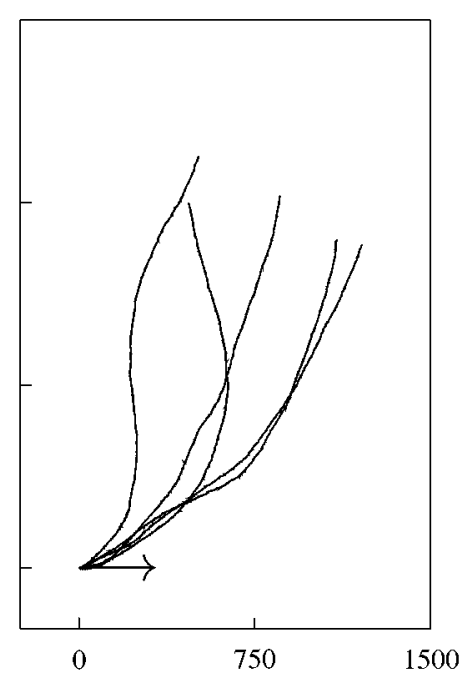

(e)

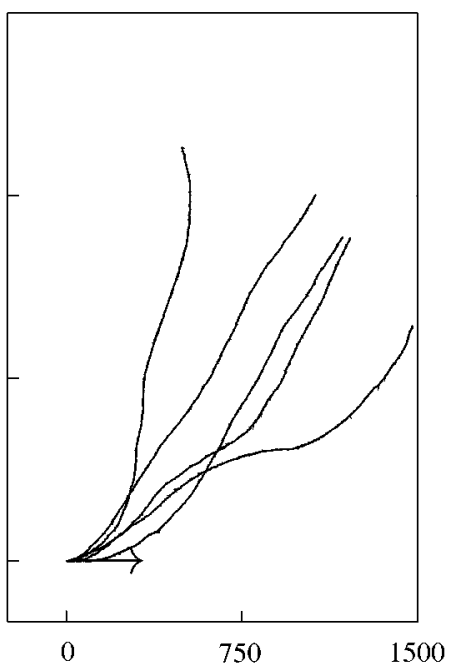

FIG. 7. Change of the slug location in time, for different gradient strengths. The location at $t=0$ is set to $(0,0)$, axes are given in grid points, and the simulations lasted for $8 \mathrm{~h}$ and $20 \mathrm{~min}$ (300000 time-steps). (a) $a_{\text {grad }}=2.5 \times 10^{-3}$; (b) $a_{\text {grad }}=1.2 \times 10^{-3}$; (c) $a_{\text {grad }}=6 \times 10^{-4}$; (d) $a_{\text {grad }}=3 \times 10^{-4}$; and (e) $a_{\text {grad }}=1.5 \times 10^{-4}$. All other parameters are as described in the legend to Fig. 2(b). For each gradient five simulations were done. In all simulations, the slug was initially positioned under an angle of $90^{\circ}$ with respect to the temperature gradient. The arrows indicate this initial orientation.

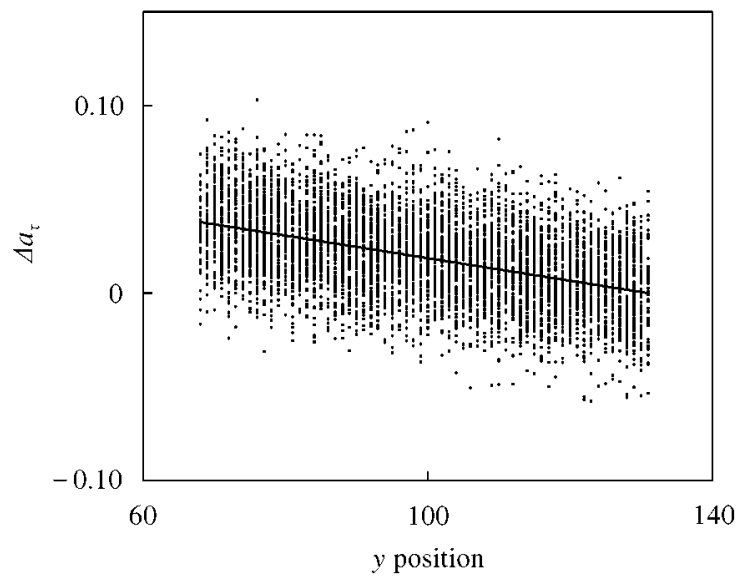

FIG. 8. Plot of $\Delta a_{\tau}$ as a function of the $y$ position at time $t=0$ for the simulations in Fig. 2(b) and (c). The solid line shows the $\Delta a_{\tau}$ distribution in the absence of noise. The dots show the values of $\Delta a_{\tau}$ for all individual automata in the presence of noise.

is used as in the previous simulation, but this time only one noise value is used for all grid points which are part of the same amoeba. This value is fixed during the whole simulation. Figure 2(d) shows such a simulation. Although the track is less stable compared to simulation in Fig. 2(c), thermotaxis is still strong. Noise levels can be increased up to 60 times the gradient strength, but at higher values of the noise S.D., different sources of cAMP waves appear, which break the slug into an increasingly higher number of ever smaller slugs.

The mechanism behind this high resistance against noise is that the cAMP wave functions as a spatio-temporal integrator. Due to the curvature effect, the cAMP wavefront is always a relatively smooth line. Differences in position are found only over longer distances. Consequently, the shape, as observed, results from the information integrated over longer time and larger distances. And since it is the chemotaxis towards this wave that gives rise to the thermotactic behaviour, global information integration becomes possible. The same process makes it possible to measure extremely small gradients, despite some internal noise generated by the model itself.

In the second experiment, with variation at the level of the individual amoebae, the variations are both temporally and spatially on a larger scale. Therefore, thermotaxis is more disturbed by this kind of noise.

\subsection{A PARTIALLY THERMOSENSITIVE SLUG}

Several researchers have stated that the process of orientation is located in the slug tip (Smith 


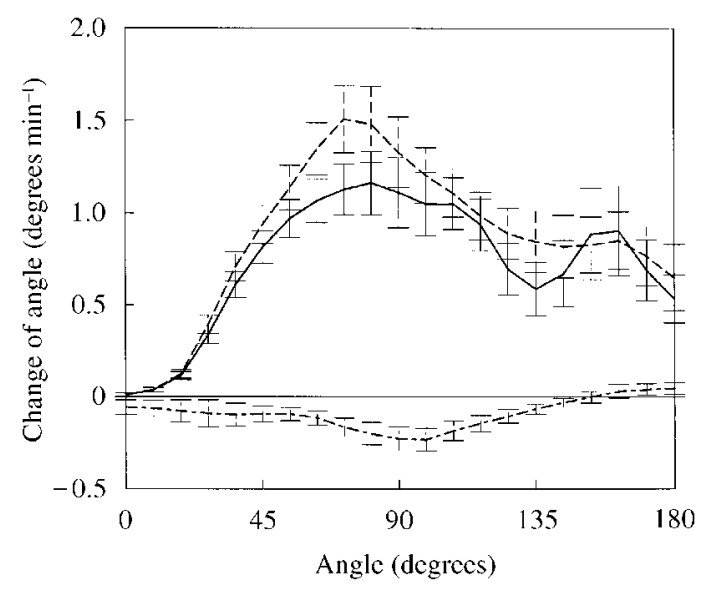

FIG. 9. Change in the angle towards the thermal gradient vs. the position the slug takes up relative to this gradient. The dashed line shows the change in the angle when only the autocycling cells in the tip of the slug are sensitive to temperature; the dashed-dotted line when only the non-autocycling cells in the body are sensitive; and the solid line when all cells are sensitive. $a_{\text {grad }}=1.2 \times 10^{-3}$, all other parameters are as described in the legend to Fig. 2(b). The means and S.E.M. (indicated by the bars) of 10 simulations per line are presented. The means and S.E.M. were calculated at intervals of $9^{\circ}$, using a Gaussian smoothing with an S.D. of again $9^{\circ}$. The change of angle is calculated using a time difference of $2 \mathrm{~min}$ 30 s. (1500 time-steps). Note that the direction of change of angle is towards the thermal gradient, i.e. the positive change means a decrease of the angle between the slug orientation and thermal gradient.

et al., 1982; Fisher, 1997). This could either indicate that there is an inherent difference between the different cell types, for example that only autocycling amoebae in the tip are thermosensitive, or that there is a functional difference between parts of the slug, i.e. all amoebae share the same thermosensitivity, but differ in behaviour solely due to their relative position inside the slug.

Figure 9 shows the velocity at which slugs turn towards the temperature gradient. The turning velocity is calculated using data from ten simulations per line, taking into account the symmetry left and right of the direction of the gradient. The figure shows the differences in the turning velocity, whether or not the tip or the rest of the slug is thermosensitive.

If the slug tip is not thermosensitive, indeed no thermotaxis whatsoever can be observed. On the other hand, desensitizing the rest of the slug has no negative effect on thermotaxis. For certain angles, the turning velocity is even slightly higher. Still, both when the whole slug is thermosensitive and when only the tip is thermosensitive, slugs turn with approximately the same velocity and the same precision towards the temperature gradient.

\section{Discussion}

\subsection{SLUG MOTION AND CELL SORTING}

We have shown that our model can reproduce important features and processes which occur in the slug, such as slug motion, cell sorting, shape conservation and the dependence of slug velocity on its mass.

In the model we have developed, the motive force is obtained by a combination of chemotaxis towards cAMP and cell adhesion (see also Savill \& Hogeweg, 1997). Instead, Williams et al. (1986) suggested that circumferential cells may squeeze and push forward the inner cell mass, while Odell \& Bonner (1986) suggested that this may be achieved by a fountain-like circulation of cells. However, fluorescence images indicated neither the circumferential movement of cells toward the inner cell mass nor active movement backward (Yumura et al., 1992).

Siegert \& Weijer (1992) showed that 3D scroll waves organize the tip. Based on these results, Bretschneider et al. (1995) made a model to describe slug motion directed by scroll waves, which is essentially the same mechanism that we use. For reasons of simplicity, we did only simulations in 2D, and therefore, such waves were not possible in our simulations. However, behind the tip, the scroll wave rapidly breaks up into planar waves, due to decreasing excitability (Dormann et al., 1996). We state that in our model we focused on these planar waves. Obviously, 3D simulations will extensively enlarge the set of possible behaviours, but the recent experiments of Bonner (1998) show that all observations of 2D slugs are consistent with what one finds in normal 3D slugs.

In all our simulations, we used a tip consisting of oscillatory amoebae, while the rest of the slug only relayed the signal. However, the change in the excitability might be much more gradual, with many more cells being in the oscillatory regime (Siegert \& Weijer, 1992; Dormann et al., 1996). Therefore, we performed a number of 
control experiments, in which all cells were oscillatory, although the tip cells were still the most excitable ones $\left(a_{t}=a_{p}=-0.05\right)$. All other conditions were kept the same. This caused no qualitative differences in any model behaviour: the high-frequency oscillations in the tip still enforce the global cAMP dynamics.

In order to obtain a stable shape of the slug, in our model, it is necessary to have a convex wave shape, which is caused by the reduced relay at the slug boundaries. In real slugs, this can be a consequence of the fact that migrating slugs are covered with slime sheath, a viscous extracellular matrix secreted by its cells. We have not explicitly modelled the slime sheath, but we interpret the diffusive loss of cAMP as being due to diffusion into this sheath. However, if we use Neumann boundary conditions, which means that there is no flux of cAMP through the boundary and hence no reduced relay, all other behaviour, such as slug motion, cell sorting and thermotaxis, are still preserved. That is, only the shape becomes inconsistent.

During the slug stage, cell redifferentiation still occurs, but it is likely to be a marginal and slow process (Schaap et al., 1996), and was left out of the model. Instead we focussed on cell sorting. In our simulations, we used $40 \%$ prestalk cells, while a more realistic value is around $25 \%$. Reducing the percentage of prestalk cells in our computations to $25 \%$ does not significantly change the results of our paper: complete cell sorting is still observed, although on a slightly longer time-scale. Hence, to present both thermotactic behaviour and cell sorting in the same time sequence, we increased the number of prestalk cells used. Cell sorting with a small amount of prestalk cells is slow due to the formation of very small clumps of prestalk cells. This is mainly a side-effect of our 2D approach, since in three dimensions larger clumps are immediately formed, as a result of the higher amount of neighbouring amoebae (Savill \& Hogeweg, 1997).

Umeda (1989) proposed a model for cell sorting, based on differential motive forces, and Bretschneider et al. (1997) also used differential chemotaxis to model sorting. However, Sternfeld (1979) found evidence that the cell sorting depends on differential cellular adhesion, and recently, Ginger et al. (1998) have found a cell surface protein which is important for both cell adhesion and cell sorting.

Sternfeld \& David (1981) found that during the process of cell sorting, cAMP is also very important. In our model, the cell sorting takes place during the cell motions that accompany the cAMP waves. We find very slow and incomplete cell sorting when we do not include the chemotactic motion towards cAMP. This is in agreement with the above finding, as well as with the results of Tasaka \& Takeuchi (1981), which show that cell sorting is facilitated by the movement of the amoebae.

MacWilliams (1982) and Meinhardt (1983) found that injecting prestalk cells into the rear part of a slug leads either to a movement of these cells towards the tip, or to the induction of a new tip, followed by the separation of a second slug. This is also in agreement with our results as described in Section 3.3.

Bonner (1998) found that the amoebae in the tip of $2 \mathrm{D}$ slugs move around in a chaotic fashion, while the posterior cells move straight ahead. In our model, we find the same behaviour: in the tip all amoebae try to move towards the source of the cAMP signal, thereby creating chaotic and swirling motion. Behind the tip the closed cAMP wave leads to straight motion.

In our model, motion is far more continuous, compared with the pulsatile cAMP signal, due to the pushing and pulling at large distances from the wavefront. The small 2D slugs that Bonner (1998) has described, also do not exhibit sharp pulsatile motion.

\subsection{THERMOTAXIS}

We have shown that thermotaxis of $D$. discoideum slugs can be explained solely by means of a temperature dependence of the cAMP-induced cAMP release and the consequences thereof for the excitability. It changes the shape of the cAMP wave, and chemotaxis towards the wave leads to the tactic behaviour: independent of the initial orientation, the slug turns towards and, afterwards, moves along the temperature gradient. The slug has a very high sensitivity, since even in the presence of extreme noise in the system (the signal/noise ratio can be as small as $1 / 300$ ), it receives the temperature signal. 
The slug behaviour in our model is due to the collective behaviour of the amoebae, as the temperature gradient is too shallow to be measured by individual amoebae. Instead, the information is first encoded as differences in the excitability.

The aim of this study was to describe the basic processes which give rise to thermotactic behaviour. We have not modelled the translation of differences in temperature into differences in excitability. We assume that this process involves chemicals such as $\mathrm{NH}_{3}$, and perhaps several other chemicals, such as slug turning factor (STF) (Fisher, 1997). In our paper, we have used a linear gradient which we normalized within the slug. In our view, such normalization is a good approximation of slug behaviour at a short time-scale. However, at longer time-scales, the relation between temperature and excitability is clearly more complicated, since both positive and negative thermotaxis are observed, as well as slow adaptations to the absolute temperature (Whitaker \& Poff, 1980). For very shallow gradients, we do not need adaptive processes (in our implementation: normalization of the gradient) in our model, for excitability can be coupled directly to the absolute temperature. However, to describe larger temperature differences an adaptive process is necessary. Instead of the normalization used here, the model could be extended with an extra equation for $a$, depending on temperature, which describes the differences in the excitability at a fast time-scale, as well as a return to the basal level at a slow time-scale. With such an extension, one could model the negative thermotaxis as well as the adaptation and compare both with observed experimental behaviour.

There has been a fair amount of dispute whether $\mathrm{NH}_{3}$ speeds up the amoebae. Therefore, we did not include an increase in velocity due to $\mathrm{NH}_{3}$ in our model. We do not state that amoebae under no conditions will have such a stronger motive force, but we show that it is not a necessary condition for thermotaxis: if there are differences in $\mathrm{NH}_{3}$, which change excitability only, thermotaxis can already be found.

Recently, Bonner (1998) has concluded that his idea of differences in the speed of motion to explain thermotaxis is not consistent with what one sees in 2D slugs. Instead, he observes that many different and changing cells are involved in pushing the tip into a different direction, which is exactly in agreement with our results.

In our model, it is mainly the tip that controls the orientation, which is in agreement with experimental findings. However, continuous cell sorting and differentiation during the slug motion, which is accompanied by ongoing changes in position, makes it unlikely that only the tip is thermosensitive, since it is observed that such a special property is not needed for thermotaxis.

A very high number of genes are in one way or another related to thermotaxis (Fisher, 1997). We did not aim to include in our model all that is known, but instead we focussed on which types of behaviour we can understand starting from some basic principles of signal transduction in $D$. discoideum. In this light, the function of all these genes might be interpreted as the tuning of the parameters to get the favoured behaviour.

This method of cell modelling lends itself excellently for describing the morphogenesis of Dictyostelium discoideum, since the behaviour observed during the developmental process are entirely driven by the local movements and responses of individual amoebae. It was used earlier to describe the process of aggregation, mount formation and slug motion (Savill \& Hogeweg, 1997), and is used here to describe a range of observed behaviours during the slug stage. By extending the model once again to $3 \mathrm{D}$, the next stages of development, as well as other facets of $D$. discoideum morphology, may be clarified.

We are grateful to Nick Savill for helpful discussions. A. F. M. Maree is supported by the Priority Program Nonlinear Systems of the Netherlands Organization for Scientific Research.

\section{REFERENCES}

BONNER, J. T. (1998). A way of following individual cells in the migrating slugs of Dictyostelium discoideum. Proc. Natl. Acad. Sci. U.S.A. 95, 9355-9359.

Bonner, J. T., HAR, D. \& Suthers, H. B. (1989). Ammonia and thermotaxis: further evidence for a central role of ammonia in the directed cell mass movements of Dictyostelium discoideum. Proc. Natl. Acad. Sci. U.S.A. 86, 2733-2736.

Bonner, J. T., Hay, A., John, D. G. \& Suthers, H. B. (1985). pH affects fruiting and slug orientation in Dictyostelium discoideum. J. Embryol. Exp. Morphol. 87, 207-213. 
Bonner, J. T., Koontz, JR, P. G. \& Paton, D. (1953). Size in relation to the rate of migration in the slime mold Dictyostelium discoideum. Mycologia 45, 235-240.

Bonner, J. T., Suthers, H. B. \& Odell, G. M. (1986). Ammonia orients cell masses and speeds up aggregating cells of slime moulds. Nature 323, 630-632.

Bretschneider, T., Siegert, F. \& WeiJer, C. J. (1995). Three-dimensional scroll waves of cAMP could direct cell movement and gene expression in Dictyostelium slugs. Proc. Natl. Acad. Sci. U.S.A. 92, 4387-4391.

Bretschneider, T., Vasiev, B. \& Weijer, C. J. (1997). A model for cell movement during Dictyostelium mound formation. J. theor. Biol. 189, 41-51.

DARCY, P. K. \& FisheR, P. R. (1990). Pharmacological evidence for a role for cyclic AMP signalling in Dictyostelium discoideum slug behaviour. J. Cell Sci. 96, 661-667.

Davies, L., SAtre, M., Martin, J.-B. \& Gross, J. D. (1993). The target of ammonia action in Dictyostelium. Cell 75, 321-327.

Dormann, D., Siegert, F. \& Weijer, C. J. (1996). Analysis of cell movement during the culmination phase of Dictyostelium development. Development 122, 761-769.

Dormann, D., VAsiev, B. \& Weijer, C. J. (1998). Propagating waves control Dictyostelium discoideum morphogenesis. Biophys. Chem. 72, 21-35.

van DuiJn, B. \& InOuYe, K. (1991). Regulation of movement speed by intracellular $\mathrm{pH}$ during Dictyostelium discoideum chemotaxis. Proc. Natl. Acad. Sci. U.S.A. 88, 4951-4955.

Feit, I. N. \& Sollitto, R. B. (1987). Ammonia is the gas used for the spacing of fruiting bodies in the cellular slime mold, Dictyostelium discoideum. Differentiation 33, 193-196.

FISHER, P. R. (1997). Genetics of phototaxis in a model eukaryote, Dictyostelium discoideum. Bioessays 19, 397407.

Ginger, R. S., Drury, L., BaAder, C., Zhukovskaya, N. V. \& Williams, J. G. (1998). A novel Dictyostelium cell surface protein important for both cell adhesion and cell sorting. Development 125, 3343-3352.

Glazier, J. A. \& Graner, F. (1993). Simulation of the differential adhesion driven rearrangement of biological cells. Phys. Rev. E 47, 2128-2154.

Grindrod, P. (1996). The Theory and Applications of Reaction-Diffusion Equations: Patterns and Waves. Oxford Applied Mathematics and Computing Science Series, 2nd Edn, Chap. 3. Oxford: Clarendon Press.

INOUYE, K. \& TAKEUCHI, I. (1979). Analytical studies on migrating movement of the pseudoplasmodium of Dictyostelium discoideum. Protoplasma 99, 289-304.

Kosugi, T. \& InOUYe, K. (1989). Negative chemotaxis to ammonia and other weak bases by migrating slugs of the cellular slime moulds. J. Gen. Microbiol. 135, 1589-1598.

MaCWilliams, H. K. (1982). Transplantation experiments and pattern mutants in cellular slime mold slugs. In: Developmental Order: its Origin and Regulation (Subtelny, S. S. \& Green, P. B., eds), pp. 463-483. New York: A. R. Liss. Martiel, J.-L. \& Goldbeter, A. (1987). A model based on receptor desensitization for cyclic AMP signaling in Dictyostelium cells. Biophys. J. 52, 807-828.

MeinhardT, H. (1983). A model for the prestalk/prespore patterning in the slug of the slime mold Dictyostelium discoideum. Differentiation 24, 191-202.
Odell, G. M. \& Bonner, J. T. (1986). How the Dictyostelium discoideum grex crawls. Phil. Trans. R. Soc. Lond. Ser. B 312, 487-525.

PANFILOV, A. V. (1991). Three dimensional vortices in active media. In: Nonlinear Wave Processes in Excitable Media (Holden, A. V., Markus, M. \& Othmer, H. G., eds), pp. 361-381. Vol. 244 of NATO ASI Series B: Physics. New York: Plenum Press.

PAnfilov, A. V. \& Pertsov, A. M. (1984). Vortex ring in three-dimensional active medium in reaction-diffusion system. Dokl. Akad. Nauk SSSR 274, 1500-1503.

SAVILL, N. J. \& HoGEWEG, P. (1997). Modeling morphogenesis: from single cells to crawling slugs. J. theor. Biol. 184, 229-235.

SchaAP, P., TANG, Y. \& OThMER, H. G. (1996). A model for pattern formation in Dictyostelium discoideum. Differentiation 60, 1-16.

SCHINDlER, J. \& SuSSMAN, M. (1979). Inhibition by ammonia of intracellular cAMP accumulation in Dictyostelium discoideum: its significance for the regulation of morphogenesis. Dev. Genet. 1, 13-20.

Siegert, F. \& WeIJER, C. J. (1992). Three-dimensional scroll waves organize Dictyostelium slugs. Proc. Natl. Acad. Sci. U.S.A. 89, 6433-6437.

Smith, E., Fisher, P. R., Grant, W. N. \& Williams, K. L. (1982). Sensory behaviour in Dictyostelium discoideum slugs: phototaxis and thermotaxis are not mediated by a change in slug speed. J. Cell Sci. 54, 329-339.

STERNFELD, J. (1979). Evidence for differential cellular adhesion as the mechanism of sorting-out of various cellular slime mold species. J. Embryol. Exp. Morph. 53, 163-178.

Sternfeld, J. \& DAvid, C. N. (1981). Cell sorting during pattern formation in Dictyostelium. Differentiation $\mathbf{2 0}$, $10-21$.

TANG, Y. \& OThMER, H. G. (1994). A G protein-based model of adaptation in Dictyostelium discoideum. Math. Biosci. 120, 25-76.

TASAKA, M. \& TAKeuchi, I. (1981). Role of cell sorting in pattern formation in Dictyostelium discoideum. Differentiation 18, 191-196.

UMEDA, T. (1989). A mathematical model for cell sorting, migration and shape in the slug stage of Dictyostelium discoideum. Bull. Math. Biol. 51, 485-500.

Weijer, C. J., McDonald, S. A. \& Durston, A. J. (1984). A frequency difference in optical-density oscillations of early Dictyostelium discoideum density classes and its implications for development. Differentiation 28, 9-12.

Whitaker, B. D. \& POFF, K. L. (1980). Thermal adaptation of thermosensing and negative thermotaxis in Dictyostelium. Exp. Cell Res. 128, 87-93.

Williams, G. B., Elder, E. M. \& Sussman, M. (1984). Modulation of the cAMP relay in Dictyostelium discoideum by ammonia and other metabolites: possible morphogenetic consequences. Dev. Biol. 105, 377-388.

Williams, K. L., Vardy, P. H. \& Segel, L. A. (1986). Cell migrations during morphogenesis: some clues from the slug of Dictyostelium discoideum. Bioessays 5, 148-152.

Yumura, S., Kurata, K. \& KitAnishi-Yumura, T. (1992). Concerted movement of prestalk cells in migrating slugs of Dictyostelium revealed by the localization of myosin. Dev. Growth Differ. 34, 319-328. 